\title{
Transformation and Differentiation of Henstock-Wiener Integrals
}

(Transformasi dan Pembezaan Kamiran Henstock-Wiener)

\author{
VARAYU BOONPOGKRONG* \& ElVIRA PEDERES DE LARA-TUPRIO
}

\section{ABSTRACT}

In this paper, transformation and differentiation of Henstock-Wiener integrals are discussed.The approach is by Riemann sums. The idea is more transparent than that of classical Wiener integral.

Keywords: Cameron-Martin theorem; Henstock integral; Henstock-Kurzweil integral; Wiener integral

\author{
ABSTRAK
}

Kertas ini membincangkan transformasi dan perbezaan kamiran Henstock-Wiener. Pendekatan yang digunakan ialah congak Riemann. Idea ini lebih telus berbanding klasik integral Wiener.

Kata kunci: Cameron-Martin theorem; Henstock integral; Henstock-Kurzweil integral; Wiener integral

\section{INTRODUCTION}

The result of transformations of Wiener integrals under translations proved by Cameron and Martin (1944) is well-known. In this paper, we shall prove this result for Henstock-Wiener integrals. Every Wiener integrable function is Henstock-Wiener integrable (Chew \& Lee 1994; Muldowney 2012, Chapter 6; Yang 1998; Yang \& Chew 1998). The Henstock-Wiener integral is defined using the Henstock-Kurzweil approach. This approach is by Riemann sums (Henstock 1988; Kurzweil 2000; Lee \& Výborný 2000). Hence the proofs of Lemma 4 and Theorem 1 are more intuitive than that of the classical Wiener integral. We also discuss differentiation of Henstock-Wiener integrals in this paper.

\section{HENSTOCK-WIENER INTEGRAL}

In this paper, denote $\prod_{t \in[0,1]} \mathbb{R}_{t}$, where $\mathbb{R}_{t}=\mathbb{R}$ for each $t$, by $\mathbb{R}^{(0,1]}$ the class of real-valued functions $\xi(t)$ defined on $[0,1]$. Let $\mathbb{R}^{(0,1]}$ be the class of all functions $\xi$ in $\mathbb{R}^{(0,1]}$ such that $\xi(0)=0$, i.e., $\mathbb{R}^{(0,1]}=\left\{\xi \in \mathbb{R}^{(0,1]}: \xi(0)=0\right\}$. Let $N$ be the class of all finite subsets of $(0,1]$.

An interval in $\mathbb{R}^{(0,1]}$, denoted by $I[N]$, is of the form

$$
I[N]=I(N) \times \mathbb{R}^{(0,1] N},
$$

where $N=\left\{t_{1}, t_{2}, \ldots, t_{n}\right\} \in \mathrm{N}$ with $t_{1}<t_{2}<\ldots<t_{n}$ and $I(N)$ is the $n$-Cartesian product of compact or unbounded closed intervals $I_{t_{i}}$ in $\mathbb{R}$, i.e., $I(N)=I_{t_{1}}, I_{t_{2}} \times \mathrm{L} \times I_{t_{n}}$.

Let $\overline{\mathbb{R}}=\mathbb{R} \cup\{\infty,-\infty\}$. Denote $\prod_{t \in[0,1]} \overline{\mathbb{R}}_{t}$ by $\overline{\mathbb{R}}^{[0,1]}$ the class of all functions $\xi(t)$ defined on $[0,1]$ with values in $\overline{\mathbb{R}}$.
Let $\overline{\mathbb{R}}^{(0,1]}$ be the class of all functions $\xi$ in $\overline{\mathbb{R}}^{[0,1]}$ such that $\xi(0)=0$, i.e., $\overline{\mathbb{R}}^{(0,1]}=\left\{\xi \in \overline{\mathbb{R}}^{[0,1]}: \xi(0)=0\right\}$. Let $\delta(\xi, N)$ be a positive function defined on $\overline{\mathbb{R}}^{(0,1]} \times \mathrm{N}$. A point-interval pair $(\xi, I[N])$, where $\xi \in \overline{\mathbb{R}}^{(0,1]}$ and $N \in \mathrm{N}$, is said to be $\delta$-fine if for each $t_{i} \in N$ : if $\xi\left(t_{i}\right) \in \mathbb{R}$, then $\xi\left(t_{i}\right) \in I_{t_{i}} \subset\left(\xi\left(t_{i}\right)\right.$ $\left.-\delta(\xi, \mathrm{N}), \xi\left(t_{i}\right)+\delta(\xi, N)\right)$; if $\xi\left(t_{i}\right)=\infty$, then $I_{t_{i}}$ is of the form $[u, \infty)$ and $I_{t_{i}} \subset\left(\frac{1}{\delta(\xi, N)}, \infty\right)$; if $\xi\left(t_{i}\right)=\infty$, then $I_{t_{i}}$ is of the form $(-\infty, v]$ and $I_{t_{i}} \subset\left(-\infty,-\frac{1}{\delta(\xi, N)}\right)$. Let $L(\xi)$ be a set-valued function defined on $\overline{\mathbb{R}}^{(0,1]}$ with values in N. Denote a pair of functions $(\delta, L)$ by $\gamma$. A point-interval pair $(\xi, I[N])$ is said to be $\gamma$-fine if $L(\xi) \subseteq N$ and $(\xi, I[N]\}$ is $\delta$-fine.

A finite collection of point-interval pairs $D=\{(\xi$, $I[N])\}$ is said to be a $\gamma$-fine partial division of $\mathbb{R}^{(0,1]}$ if $\{I[N]\}$ is a partial partition of $\mathbb{R}^{(0,1]}$, that is, the collection $\{I[N]\}$ is non-overlapping and their union is a subset of $\mathbb{R}^{(0,1]}$, and each $(\xi, I[N])$ is $\gamma$-fine. In addition, if $\{I[N]\}$ is a partition of $\mathbb{R}^{(0,1]}$, then $D$ is said to be a $\gamma$-fine division of $\mathbb{R}^{(0,1]}$. It is known that given a function $\gamma$, a $\gamma$-fine division of $\mathbb{R}^{(0,1]}$ exists, see (Muldowney 2012, p.121).

Given $N=\left\{t_{1}, t_{2}, \ldots, t_{n}\right\} \in \mathrm{N}$ with $t_{1}<t_{2}<\ldots<t_{n}$, let

$$
G(I[N])=\int_{I(N)} h_{N}(u) d u,
$$

where $u=\left(u_{1}, u_{2}, \ldots, u_{n}\right)$ and

$$
h_{N}(u)=\left((2 \pi)^{n} \prod_{j=1}^{n}\left(t_{j}-t_{j-1}\right)\right)^{-1 / 2} \exp \left(-\frac{1}{2} \sum_{j=1}^{n} \frac{\left(u_{j}-u_{j-1}\right)^{2}}{t_{j}-t_{j-1}}\right)
$$


with $t_{0}=0$ and $u_{0}=0$. The $n$-dimensional integral above is Riemann or improper Riemann integral. Hence, it is a Henstock integral, see (Henstock 1988; Lee \& Výborný 2000; Muldowney 2012). The integral function $G(I[N])$ is the Wiener measure of the interval $I(N)$ in $\mathbb{R}^{(0,1]}$, which is equal to the Wiener measure of the interval $I(N)$ in the $n$-dimensional space. For $\xi \in \overline{\mathbb{R}}^{(0,1]}$, let

$$
g(\xi, I[N])=h_{N}(\xi(N))|I(N)|,
$$

where $\left.\xi(N)=\left(\xi\left(t_{1}\right), \xi\left(t_{2}\right), \ldots, \xi\left(t_{n}\right)\right), \mid I(N)\right) \mid$, is the volume of $I(N)$ defined by $|I(N)|=\prod_{i=1}^{n} \mid I_{t_{i}}$, in which $\left|I_{t_{i}}\right|=v-\mathrm{u}$ if $I_{t_{i}}$ is of the form $[u, v]$ and $I_{t_{i}}=\infty$ if $I_{t_{i}}$ is unbounded. The function $g(\xi, I[N])$ is an approximate Wiener measure of the interval $I[N]$, Lemma 2 . Note that, given a function $\gamma$, $(\xi, I[N])$ is $\gamma$-fine, when $I(N)$ is unbounded and $h_{N}(\xi(N))$ $=0$ when $\xi(N)$ has infinite components.

Definition 1 (Henstock-Wiener Integral on $\mathbb{R}^{(0,1]}$ ) The function $f: \overline{\mathbb{R}}^{(0,1]} \rightarrow \mathbb{R}$ is said to be Henstock-Wiener integrable to $A \in \mathbb{R}$ on $\mathbb{R}^{(0,1]}$ if for each $\epsilon>0$, there exists a pair of functions $\gamma=(\delta, L)$ such that whenever $D=\{(\xi$, $I[N])\}$ is a $\gamma$-fine division of $\mathbb{R}^{(0,1]}$, we have

$$
\left|(D) \sum f(\xi) G(I[N])-A\right| \leq \epsilon,
$$

where we assume that $f(\xi)=0$ if one of the components of $\xi$ is $\pm \infty$. The number $A$ is called the Henstock-Wiener integral of $f$ on $\mathbb{R}^{(0,1]}$ and is denoted by $\int_{\mathbb{R}(0,1]} f$.

We note that the basic properties of integrals, such as linear property and the integrability over subinterval hold for the Henstock-Wiener Integral on $\mathbb{R}^{(0,1]}$.

If $H \subseteq \mathbb{R}^{(0,1]}$, then the Henstock-Wiener integral of $f$ on $H$, denoted by $\int_{H} f$, is defined by $\int_{H} f=\int_{\mathbb{R}(0,1]} f \chi_{H}$, where $\chi_{H}(\xi)=1$ if $\xi \in H$ and 0 otherwise.

Note that when $f=1$, then $\int_{\mathbb{R}(0,1]} f=1$, (Muldowney 2012, p. 285) and $\int_{C} f=1$, where $C$ is the space of all functions $\xi(t)$ continuous on $[0,1]$ with $\xi(0)=0$, see (Muldowney 1987, p. 64-65; Muldowney 2012, Section 6.9, p.288; Yang 1998, p. 49).

Lemma 1 (Henstock's Lemma) (Muldowney 2012, Theorem 18,p. 132) Let $f: \overline{\mathbb{R}}^{(0,1]} \rightarrow \mathbb{R}$ be Henstock-Wiener integrable on $\mathbb{R}^{(0,1]}$. Then, for each $\epsilon>0$, there exists a pair of functions $\gamma=(\delta, L)$ such that whenever $D=\{(\xi, I[N])\}$ is a $\gamma$-fine division of $\mathbb{R}^{(0,1]}$, we have

$$
\text { (D) } \sum\left|f(\xi) G(I[N])-\int_{I[N]} f\right| \leq \epsilon .
$$

As mentioned earlier, $g(\xi, I[N])$ can be used to approximate the Wiener measure $G(I[N])$ of $I[N]$. The difference $|g(\xi, I[N])-G(I[N])|$ represents the error of this approximation. The following lemma is analogous to the Henstock Lemma, which says that the accumulated error is small. The result will then allow us to use henceforth the approximate function $g(\xi, I[N])$ instead of $G(I[N])$.
Lemma 2 (Muldowney et al. 2012, p. 285) The functions $g(\xi, I[N])$ and $G(I[N])$ are variationally equivalent, i.e., for each $\epsilon>0$, there exists a pair of functions $\gamma=(\delta, L)$ such that whenever $D=\{(\xi, I[N])\}$ is a $\gamma$-fine division of $\mathbb{R}^{(0,1]}$, we have

$$
\text { (D) } \sum|g(\xi, I[N\})-G(I[N])| \leq \epsilon
$$

\section{TRANSLATION}

First we state the Cameron-Martin Theorem, which is a translation theorem.

Theorem 1 (Cameron-Martin Theorem) Let $\eta \in C$ be differentiable with its derivative $\eta$ ' of bounded variation on $[0,1]$. Then $f$ is Henstock-Wiener integrable on $C$ if and only if $f_{\eta} \kappa_{\eta}$ is Henstock-Wiener integrable on $C$, where $f_{\eta}(\xi)=f(\xi+\eta)$. Moreover,

$$
\int_{C} f(\xi)=\int_{C} f(\xi+\eta) \kappa_{\eta}(\xi),
$$

where

$$
\kappa_{\eta}(\xi)=\exp \left(-\frac{1}{2} \int_{0}^{1}\left(\eta^{\prime}(t)\right)^{2} d t-\int_{0}^{1} \eta^{\prime}(t) d \xi(t)\right)
$$

if $\xi \in C$, and $\kappa_{\eta}(\xi)=0$ if $\xi \in \overline{\mathbb{R}}^{(0,1]} \backslash C$.

The theorem will be proved by established four lemmas, Lemma 2 to 5. Recall first that the HenstockWiener integral is defined by Riemann sums of the form $\sum f(\xi) G(I[N])$. The previous lemma, Lemma 2, says that $G(I[N])$ can be approximated by $g(\xi, I[N])$ therefore, we can make use of the latter instead. Next, by straightforward calculation, Lemma 3 shows that the translation $\mathrm{g}(\xi+\eta$, $I[N]+\eta)$ of $\mathrm{g}(\xi, I[N])$ under the function $\eta$ is given by $g(\xi, I[N]) \cdot \tau \eta(\xi, N)$, where $\tau$ is defined in (2.2). Moreover, Lemma 4 shows that the continuous version $\kappa_{\eta}(\xi)$ can be approximated by the discrete version $\tau_{\eta}(\xi, N)$. Finally, Lemma 5 states that the continuous version $g_{\eta}^{\kappa}$ can be approximated by the discrete version $g_{\eta}^{\tau}$.

We shall now give details of the proofs. First, for any fixed $\eta \in \mathbb{R}^{(0,1]}$, we shall find the translation $g(\xi+\eta, I[N]$ $+\eta$ ) of $g$ under $\eta$, (Lemma 3 ). In relation to the definition of $h_{N}(\xi(N))$ in (1.1), let $\tau_{\eta}: \mathbb{R}^{(0,1]} \times \mathcal{N} \rightarrow \mathbb{R}$ be defined by

$$
\begin{aligned}
\tau_{\eta}(\xi, N)= & -\frac{1}{2} \sum_{j=1}^{n} \frac{\left(\eta\left(t_{j}\right)-\eta\left(t_{j-1}\right)\right)^{2}}{t_{j}-t_{j-1}} \\
& \left.-\sum_{j=1}^{n} \frac{\left(\xi\left(t_{j}\right)-\xi\left(t_{j-1}\right)\right)\left(\eta\left(t_{j}\right)-\eta\left(t_{j-1}\right)\right)}{t_{j}-t_{j-1}}\right)
\end{aligned}
$$

and let $g_{\eta}^{\tau}(\xi, I[N])=g(\xi, I[N]) \cdot \tau_{\eta}(\xi, N)=h_{N}(\xi(N)) \mid \cdot \tau_{\eta}(\xi$, $N)$ when $\xi \in \mathbb{R}^{(0,1]}$, and $g_{\eta}^{\tau}(\xi, I[N])=0$ when $\xi$ has infinite components. 
Lemma 3 Let $\eta \in \mathbb{R}^{(0,1]}$. Then for any $\xi \in \mathbb{R}^{(0,1]}$, we have

$$
g(\xi+\eta, I[N]+\eta)=g_{\eta}^{\tau}(\xi, I[N]) .
$$

Proof. This is a straightforward calculation.

$$
\begin{aligned}
g(\xi+\eta, I[N]+\eta) & =h_{N}((\xi+\eta)(N))|I(N)+\eta| \\
& =h_{N}((\xi+\eta)(N))|I(N)| \\
& =h_{N}(\xi(N)) \cdot \tau_{\eta}(\xi, N)|I(N)| \\
& =g(\xi, I[N]) \cdot \tau_{\eta}(\xi, N) \\
& =g_{\eta}^{\tau}(\xi, I[N]) .
\end{aligned}
$$

Let $\eta \in C$ be differentiable and let the derivative of $\eta$ be of bounded variation on $[0,1]$. Then $\left(\eta^{\prime}\right)^{2}$ is Riemann integrable on $[0,1]$. Moreover, if $\xi \in C$, then $\eta^{\prime}$ is Riemann-Stieltjes integrable with respect to $\xi$ on $[0,1]$, (Apostol 1957, p.211; Henstock 1988, p.7). Note that, in (2.2), the function $\tau_{\eta}(\xi, N)$ is a discrete version using the points in $N$, while the corresponding continuous version $\kappa_{\eta}(\xi)$ is given in (2.1). Lemma 4 affirms that we can use the discrete version $\tau_{\eta}(\xi, N)$ to approximate the coutinuous version $\kappa_{\eta}(\xi)$ and the error is small when $N$ is big enough.

Lemma 4 Let $\eta \in C$ be differentiable with its derivative $\eta^{\prime}$ of bounded variation on $[0,1]$, and let $\xi \in C$. Then there exists $L_{\eta}(\xi) \in \mathcal{N}$ such that for any $N \in \mathcal{N}$ with $L_{\eta}(\xi) \subseteq$ $N$, we have

$$
\left|\tau_{\eta}(\xi, N)-\kappa_{\eta}(\xi)\right| \leq \epsilon
$$

Proof. Let $\epsilon_{1}>0$ be given. Since $\left(\eta^{\prime}\right)^{2}$ is Riemann integrable on $[0,1]$ and $\eta$ ' is Riemann-Stieltjes integrable with respect to $\xi$ on $[0,1]$, there exists a partition $P$ of $[0,1]$ such that for any refinement partition $Q=\{[s, w]\}$ of $P$, we have

$$
\left|(Q) \sum\left(\eta^{\prime}(x)\right)^{2}(w-s)-\int_{0}^{1}\left(\eta^{\prime}(t)\right)^{2} d t\right| \leq \frac{\epsilon_{1}}{2}
$$

and

$$
\left|(Q) \sum \eta^{\prime}(x)(\xi(w)-\xi(s))-\int_{0}^{1} \eta^{\prime}(t) d \xi(t)\right| \leq \frac{\epsilon_{1}}{2},
$$

where $x$ is any point in $[s, w]$.

By Mean Value Theorem, for each interval $[s, w]$, there exists $y \in[s, w]$ such that

$$
\eta^{\prime}(y)=\frac{\eta(w)-\eta(s)}{w-s}
$$

Thus, by inequality (2.3), we have

$$
\begin{aligned}
\mid(Q) & \sum \frac{(\eta(w)-\eta(s))^{2}}{w-s}-\int_{0}^{1}\left(\eta^{\prime}(t)\right)^{2} d t \mid \\
& =\left|(Q) \sum\left(\eta^{\prime}(y)\right)^{2}(w-s)-\int_{0}^{1}\left(\eta^{\prime}(t)\right)^{2} d t\right| \leq \frac{\epsilon_{1}}{2}
\end{aligned}
$$

and, by inequality (2.4), we have

$$
\begin{gathered}
\mid(Q) \sum \frac{(\eta(w)-\eta(s))(\xi(w)-\xi(s))}{w-s}-\int_{0}^{1} \eta^{\prime}(t) d \xi(t) \\
=\left|(Q) \sum\left(\eta^{\prime}(y)\right)^{2}(w-s)-\int_{0}^{1}\left(\eta^{\prime}(t)\right)^{2} d t\right| \leq \frac{\epsilon_{1}}{2}
\end{gathered}
$$

Suppose the partition $P$ is induced by $\left\{t_{0}, t_{1}, \ldots, t_{n}\right\}$ with $0=t_{0}<t_{1}<\ldots<t_{n}=1$. Let $L_{\eta}(\xi)=\left\{t_{1}, \ldots, t_{n}\right\}$.

Let $\epsilon>0$ be given. By the continuity of $\exp (z)$ at $-\frac{1}{2} \int_{0}^{1}\left(\eta^{\prime}(t)\right)^{2} d t-\int_{0}^{1} \eta^{\prime}(t) d \xi(t)$, there exists $\epsilon_{1}>0$ such that whenever inequalities (2.5) and (2.6) hold, we have

$$
\left|\tau_{\eta}(\xi, N)-\kappa_{\eta}(\xi)\right| \leq \epsilon
$$

when $N \in \mathcal{N}$ with $L_{\eta}(\xi) \subseteq N$.

Let $g_{\eta}^{\kappa}(\xi, I[N])^{\eta}=g(\xi, I[N]) \cdot \kappa_{\eta}(\xi)$. By the above result, now we can use the discrete version $g_{\eta}^{\tau}(\xi, I[N])$ to approximate the continuous version $g_{\eta}^{\kappa}(\xi, I[N])$. Lemma 5 says that the accumulated error is small, which is analogous to the result of Henstock Lemma.

Lemma 5 Let $\eta \in C$ be differentiable with its derivative $\eta$ ' of bounded variation on $[0,1]$. Then $g_{\eta}^{\tau}(\xi, I[N])$ and $g_{\eta}^{\kappa}$ $(\xi, I[N])$ are variationally equivalent, i.e., for each $\epsilon>0$, there exists a pair offunctions $\gamma=(\delta, L)$ such that whenever $D=\{(\xi, I[N])\}$ is a $\gamma$-fine division of $\mathbb{R}^{(0,1]}$, we have

$$
\text { (D) } \sum\left|g_{\eta}^{\tau}(\xi, I[N])-g_{\eta}^{\kappa}(\xi, I[N])\right| \leq \epsilon .
$$

Proof. Let $\epsilon>0$ be given. Since, by Lemma 2, $g(\xi, I[N])$ and $G(I[N])$ are variationally equivalent, there exists a pair of functions $\gamma_{1}=\left(\delta_{1}, L_{1}\right)$ such that whenever $D_{1}=$ $\{(\xi, I[N])\}$ is a $\gamma_{1}$-fine division of $\mathbb{R}^{(0,1]}$, we have

$$
\left(D_{1}\right) \sum|g(\xi, I[N])-G(I[N])| \leq \epsilon .
$$

Let $\delta(\xi, N)=\delta_{1}(\xi, N)$ for all $\xi \in \overline{\mathbb{R}}^{(0,1]}$ and $N \in \mathrm{N}$. Let $L(\xi)=L_{1}(\xi) \cup L_{\eta}(\xi)$ for all $\xi \in C$, where $L_{\eta}(\xi)$ is as defined in Lemma 4 and $L(\xi)=L_{1}(\xi)$ when $\xi \in \mathbb{R}^{(0,1]} \backslash C$. Choose $\gamma=(\delta, L)$. Let $D=\{(\xi, I[N])\}$ be a $\gamma$-fine division of $\mathbb{R}^{(0,1]}$. Then

$$
\begin{aligned}
& \text { (D) } \sum\left|g_{\eta}^{\tau}(\xi, I[N])-g_{\eta}^{\kappa}(\xi, I[N])\right|= \\
& (D) \sum\left|\tau_{\eta}(\xi, N)-\kappa_{\eta}(\xi)\right| g(\xi, I[N]) \\
& \leq(D) \sum \epsilon g(\xi, I[N]) \\
& =\epsilon\left((D) \sum|g(\xi, I[N])-G(I[N])|+(D) \sum G(I[N])\right) \\
& \leq \epsilon\left(\epsilon+(D) \sum G(I[N])\right) .
\end{aligned}
$$

Since there exists a constant $B$ such that for any $D \sum G(I[N]) \leq B$, see (Muldowney 2012, p. 285, Theorem $168)$, then we get the desired result. 
Let $f: \mathbb{R}^{(0,1]} \rightarrow \mathbb{R}$ and $\eta \in \mathbb{R}^{(0,1]}$ be given. Define

$$
\rho(\xi)=f(\xi+\eta) .
$$

Note that, similar to $f$, we may extend the domain of $\rho$ by assuming that $\rho(\xi)=0$ when $\xi$ has infinite components.

Proof of Theorem 1. Let $\epsilon>0$ be given. Suppose $f$ is Henstock-Wiener integrable on $C$. Then there exists a pair of functions $\gamma_{1}=\left(\delta_{1}, L_{1}\right)$ such that whenever $\bar{D}=\{(\xi$, $I[N])\}$ is a $\gamma_{1}$-fine division of $\mathbb{R}^{(0,1]}$, we have

$$
\left|(\bar{D}) \sum\left(f \cdot \chi_{C}\right)(\xi) G(I[N])-\int_{C} f\right| \leq \frac{\epsilon}{2} .
$$

By Lemma 2, we may assume that for such $\bar{D}$

$$
\left|(\bar{D}) \sum\left(f \cdot \chi_{C}\right)(\xi) g(\xi, I[N])-\int_{C} f\right| \leq \frac{\epsilon}{2} .
$$

Let $\delta_{1_{\eta}}: \overline{\mathbb{R}}^{(0,1]} \times \mathrm{N} \rightarrow \mathbb{R}$ be defined by $\delta_{1_{\eta}}(\xi, N)=$ $\delta_{1}(\xi+\eta, N)$ for all $\xi \in \overline{\mathbb{R}}^{(0,1]}$ and $N \in \mathrm{N}$ and $L_{1_{\eta}}: \overline{\mathbb{R}}^{(0,1]}$ $\rightarrow \mathrm{N}$ defined by $\mathrm{L}_{1_{\eta}}(\xi)=L_{1}(\xi+\eta)$ for all $\xi \in \overline{\mathbb{R}}^{(0,1]}$.

Let

$$
H_{k}=\left\{x \in \overline{\mathbb{R}}^{(0,1]}: k-1 \leq \mid \rho<k\right\},
$$

for $k=1,2, \ldots$. Recall that $\rho(x)=f(x+\eta)$.

By Lemma $5, g_{\eta}^{\tau}(\xi, I[N])$ and $g_{\eta}^{\kappa}(\xi, I[N])$ are variationally equivalent. That is, there exists a pair of functions $\gamma_{2_{\eta}}=\left(\delta_{2_{\eta}}, L_{2_{\eta}}\right)$ such that whenever $D_{k}=\{(\zeta$, $J[N])\}$ is a $\gamma_{{ }_{2 \eta}}$-fine partial division of $\mathbb{R}^{(0,1]}$ with $\zeta \in H_{k}$, we have

$$
\left(D_{k}\right) \sum\left|g_{\eta}^{\tau}(\zeta, J[N])-g_{\eta}^{\kappa}(\xi, J[N])\right| \leq \frac{\epsilon}{k 2^{k+1}} .
$$

Let $\delta_{\eta}: \overline{\mathbb{R}}^{((0,]} \times \mathrm{N} \rightarrow \mathbb{R}$ be defined by $\delta_{\eta}(\xi, N)=\min$ $\left\{\delta_{2_{\eta}}(\xi, N)\right\}$ for all $\xi \in \overline{\mathbb{R}}^{(0,1]}$ and $N \in \mathrm{N}$, and $L_{\eta}: \overline{\mathbb{R}}^{(0,1]} \rightarrow \mathrm{N}$ defined by $L_{\eta}(\xi)=L_{1_{\eta}}(\xi) \cup L_{2_{\eta}}(\xi)$ for all $\xi \in \overline{\mathbb{R}}^{(0,1]}$. Choose $\gamma_{\eta}=\left(\delta_{\eta}, L_{\eta}\right)$. Let $\left.D_{\eta}=\{\zeta, J[N])\right\}$ be a $\gamma_{\eta}$-fine division of $\mathbb{R}^{(0,1]}$. Thus, by inequality (2.9), we have

$$
\begin{aligned}
\mid\left(D_{\eta}\right) & \sum\left(\rho \cdot \chi_{C}\right)(\zeta)\left[g_{\eta}^{\tau}(\xi, J[N])-g_{\eta}^{\kappa}(\zeta, J[N)]\right. \\
& \leq\left(D_{\eta}\right) \sum\left|\left(\rho \cdot \chi_{C}\right)(\zeta)\right|\left|g_{\eta}^{\tau}(\zeta, J[N])-g_{\eta}^{\kappa}(\zeta, J[N])\right| \\
& =\sum_{k=1}^{\infty}\left(D_{k}\right) \sum\left|\left(\rho \cdot \chi_{H_{k}}\right)(\xi)\right|\left|g_{\eta}^{\tau}(\zeta, J[N])-g_{\eta}^{\kappa}(\zeta, J[N])\right| \\
& <\sum_{k=1}^{\infty} k \cdot\left(D_{k}\right) \sum\left|g_{\eta}^{\tau}(\xi, J[N])-g_{\eta}^{\kappa}(\zeta, J[N])\right| \\
& \leq \sum_{k=1}^{\infty} k \cdot \frac{\epsilon}{k 2^{k+1}}=\frac{\epsilon}{2} .
\end{aligned}
$$

Let $\xi=\xi+\eta$ and $I[N]=J[N]+\eta$. Then $D=\{\xi, I[N])\}$ is a $\gamma_{1}$-fine division of $\mathbb{R}^{(0,1]}$. Then, by inequality $(2.8)$ and Lemma 3, we have

$$
\begin{aligned}
& \left|\left(D_{\eta}\right) \sum\left(\rho \cdot \chi_{C}\right)(\xi) g_{\eta}^{\tau}(\zeta, J[N])-\int_{C} f\right|= \\
& \left|\left(D_{\eta}\right) \sum\left(f \cdot \chi_{C}\right)(\xi+\eta) g(\xi+\eta, J[N]+\eta)-\int_{C} f\right| \\
& =\left|(D) \sum\left(f \cdot \chi_{C}\right)(\xi) g(\xi, I[N])-\int_{C} f\right| \\
& \leq \frac{\epsilon}{2} .
\end{aligned}
$$

By inequalities (2.10) and (2.11), we have

$$
\begin{aligned}
& \left|\left(D_{\eta}\right) \sum\left(\rho \cdot \chi_{C}\right)(\xi) \kappa_{\eta}(\xi) g(\xi, J[N])-\int_{C} f\right|= \\
& \left|\left(D_{\eta}\right) \sum\left(\rho \cdot \chi_{C}\right)(\xi) g_{\eta}^{\kappa}(\xi, J[N])-\int_{C} f\right| \\
& \leq\left|\left(D_{\eta}\right) \sum\left(\rho \cdot \chi_{C}\right)(\xi) g_{\eta}^{\tau}(\xi, J[N])-\int_{C} f\right| \\
& +\mid\left(D_{\eta}\right) \sum\left(\rho \cdot \chi_{C}\right)(\zeta)\left[g_{\eta}^{\tau}(\zeta, J[N])-g_{\eta}^{\kappa}(\zeta, J[N)]\right. \\
& \leq \frac{\epsilon}{2}+\frac{\epsilon}{2}=\epsilon
\end{aligned}
$$

Therefore, $\rho_{\kappa_{\eta}}$ is Henstock-Wiener integrable on $C$ and

$$
\int_{C} \rho \kappa_{\eta}=\int_{C} f \text {. }
$$

The proof for the converse is analogous. Hence, we get the required result.

\section{DIFFERENTIATION OF HENSTOCK-WIENER INTEGRAL}

Let $I$ be the collection of all intervals in $\mathbb{R}^{(0,1]}$. In the following definition, $G$ is the function defined by (1.1).

Definition 2 (Henstock-Wiener derivative on $\mathbb{R}^{(0,1]}$ ) The function $F: I \rightarrow \mathbb{R}$ is said to be Henstock-Wiener differentiable at $\xi \in \mathbb{R}^{(0,1]}$ with derivative $f(\xi)$ if for each $\epsilon>0$, there exists a pair of functions $\gamma=(\delta, L)$ such that whenever $(\xi, I[N]), \xi \in \overline{\mathbb{R}}^{(0,1]}$, is $\gamma$-fine, we have

$$
|F(I[N])-f(\xi) G(I[N])| \leq \epsilon G(I[N]) .
$$

Let $f: \overline{\mathbb{R}}^{(0,1]} \rightarrow \mathbb{R}$ and $F: \mathrm{I} \rightarrow \mathbb{R}$ be additive. Given $\epsilon>0$, define

$$
\Gamma_{\rho}=\{(\xi, I[N]): \xi \in I[N]
$$

and

$$
|F(I[N])-f(\xi) G(I[N])|>\epsilon G(I[N])\} .
$$

Note that $\Gamma_{\epsilon}$ is the collection of point-interval pairs that do not satisfy inequality (3.1). Such collection plays an important role in differentiation.

For each $k, k=1,2 \ldots$, and each pair of functions $\gamma=$ $(\delta, L)$, let $h(\xi, I[N])=f(\xi) G(I[N])$, 


$$
\begin{aligned}
I V\left(h, \Gamma_{1 / k}, \gamma\right)= & \sup _{D}\left\{(D) \sum h(\xi, I[N]) \mid: D \subseteq \Gamma_{1 / k}\right. \\
& \text { is } \left.\gamma \text {-fine partial division of } \mathbb{R}^{(0,1]}\right\}, \\
I V\left(F, \Gamma_{1 / k}, \gamma\right)= & \sup _{D}\left\{(D) \sum|F(I[N])|: D \subseteq \Gamma_{1 / k}\right. \\
& \text { is } \left.\gamma \text {-fine partial division of } \mathbb{R}^{(0,1]}\right\}
\end{aligned}
$$

and

$$
\begin{aligned}
& I V\left(h, \Gamma_{1 / k}\right)=\inf _{\gamma} I V\left(h, \Gamma_{1 / k}, \gamma\right) \text { and } \\
& I V\left(F, \Gamma_{1 / k}\right)=\inf _{\gamma} I V\left(F, \Gamma_{1 / k}, \gamma\right) .
\end{aligned}
$$

$I V\left(h, \Gamma_{1 / k}\right)$ and $I V\left(F, \Gamma_{1 / k}\right)$ are called the variations of $h$ and $F$ in $\Gamma_{1 / k}$, respectively (Henstock 1988,p.54). The important idea here is that we do not discuss the differentiability at a point. We focus on those point-interval pairs $(\xi, I[N])$ that do not satisfy the inequality (3.1) of differentiation, which plays an important role in differentiation, i.e., those $(\xi, I[N])$ in $\Gamma_{1 / k}$. We say that the derivative of the interval function $F(I[N])$ is equal to the point function $f(\xi)$ for almost all point-interval pairs $(\xi, I[N])$ in $\mathbb{R}^{(0,1]} \times \mathrm{I}$ if $I V\left(h, \Gamma_{1 / k}\right)=I V\left(F, \Gamma_{1 / k}\right)=0$ for $k=1,2, \ldots$. In this case we write $F^{\prime}(I[N])=f(\xi)$ almost all in $\mathbb{R}^{(0,1]} \times \mathrm{I}$.

In this section, we shall show that if $f$ is HenstockWiener integrable on $\mathbb{R}^{(0,1]}$ with primitive $F$, then the derivative $F^{\prime}(I[N])$ of $F(I[N])$ exists and $F^{\prime}(I[N])=f(\xi)$ almost all $(\xi, I[N])$ (Theorem 4$)$. To prove the converse of the above result, we need to consider $\xi \in \overline{\mathbb{R}}^{(0,1]}, \mathbb{R}^{(0,1]}$. Therefore we need the following definition of $A C$.

Let $F: \mathrm{I} \rightarrow \mathbb{R}$ be additive. The interval function $F$ is said to be $A C$ near infinity if for each $\epsilon>0$, there exists a pair of functions $\gamma=(\delta, L)$ such that whenever $D=\{(\xi, I[N])\}$, $\xi \in \overline{\mathbb{R}}^{(0,1]}, \mathbb{R}^{(0,1]}$, is a $\gamma$-fine partial division of $\mathbb{R}^{(0,1]}$, we have

$$
\text { (D) } \sum|F(I[N])| \leq \epsilon
$$

We remark that the primitive function $F$ of a Henstock-Wiener integrable function $f$ is $A C$ near infinity by Henstock's Lemma.

First we shall prove a result of differentiation using double Lusin condition (3.2).

Theorem 2 Let $f: \overline{\mathbb{R}}^{(0,1]} \rightarrow \mathbb{R}$ and $F: \mathrm{I} \rightarrow \mathbb{R}$ be additive. Then $f$ is Henstock-Wiener integrable on $\mathbb{R}^{(0,1]}$ with primitive $F$ if and only if $F$ is $A C$ near infinity and for any $\epsilon>0$, for each $k=1,2, \ldots$, there exists a pair of functions $\gamma_{k}=\left(\delta_{k}\right.$, $\left.L_{k}\right)$ such that whenever $D=\{(\xi, I[N])\} \subseteq \Gamma_{1 / k}$ is a $\gamma_{k}$-fine partial division of $\mathbb{R}^{(0,1]}$, we have

(D) $\Sigma|f(\xi) G(I[N])| \leq \grave{U}$ and $(D) \sum|F(I[N])| \leq \epsilon$.
Proof The following proof follows the ideas of Cabral et al. (2000-2001); Lu and Lee (1999).

Let $0<\rho<1$ be given. Suppose $f$ is Henstock-Wiener integrable on $\mathbb{R}^{(0,1]}$ with primitive $F$. Let

$$
H_{m}=\left\{x \in \mathbb{R}^{(0,1]}: m-1 \leq|f(x)|<m\right\}
$$

for $m=1,2, \ldots$ By Henstock's Lemma (Lemma 1), for each $m$ and $k, m, k=1,2, \ldots$, there exists a pair of functions $\gamma_{m, k}=\left(\delta_{m, k}, L_{m, k}\right)$ such that whenever $D=\{(\xi, I[N])\}$ is a $\gamma_{m, k}$-fine division of $\mathbb{R}^{(0,1]}$, we have

$$
I V\left(h,(D) \sum|F(I[N])-f(\xi) G(I[N])| \leq \frac{\epsilon}{m k 2^{m+1}} .\right)
$$

For each $\xi \in \mathbb{R}^{(0,1]}$ and $N \in \mathrm{N}$, define $\delta_{k}(\xi, N)=$ $\delta_{m, k}(\xi, N)$ and $L_{k}(\xi)=L_{m, k}(\xi)$ if $\xi \in H_{m}$. Consider the pair of functions $\gamma_{k}=\left(\delta_{k}, L_{k}\right)$ and let $D=\{(\xi, I[N])\} \subseteq \Gamma_{1 / k}$ be a $\gamma_{k}$-fine partial division of $\mathbb{R}^{(0,1]}$. For each $m$, let $D_{m} \stackrel{1 / k}{=}\{(\xi$, $\left.I[N]) \in D: \xi \in H_{m}\right\}$. Then

$$
\begin{aligned}
(D) \sum|f(\xi) G(I[N])| & =\sum_{m=1}^{\infty}\left(D_{m}\right) \sum|f(\xi)| G(I[N]) \\
& <\sum_{m=1}^{\infty} m \cdot\left(D_{k}\right) \sum \frac{|F(I[N])-f(\xi) G(I[N])|}{1 / k} \\
& \leq \sum_{m=1}^{\infty} m \cdot \frac{\epsilon}{m 2^{m+1}}=\frac{\epsilon}{2} .
\end{aligned}
$$

Hence,

$$
\begin{aligned}
(D) \sum|F(I[N])| & \leq(D) \sum|F(I[N])-f(\xi) G(I[N])| \\
& +(D) \sum|f(\xi) G(I[N])| \\
& \leq \sum_{m=1}^{\infty}\left(D_{m}\right) \sum|F(I[N])-f(\xi) G(I[N])|+\frac{\epsilon}{2} \\
& \leq \sum_{m=1}^{\infty} \frac{\epsilon}{m k 2^{k+1}}+\frac{\epsilon}{2} \leq \sum_{m=1}^{\infty} \frac{\epsilon}{2^{m+1}}+\frac{\epsilon}{2}=\frac{\epsilon}{2}+\frac{\epsilon}{2}=\epsilon .
\end{aligned}
$$

Conversely, let $\epsilon>0$ and $k=1,2, \ldots$ be given, suppose there exists a pair of functions $\gamma_{k}=\left(\delta_{k}, L_{k}\right)$ such that whenever $D=\{(\xi, I[N])\} \subseteq \Gamma_{1}$ is a $\gamma_{k}$-fine partial division of $\mathbb{R}^{(0,1]}$, inequalities (3.2) hold and $\left(D_{\infty}\right) \Sigma \mid F(I[N])$ $\mid \leq \epsilon$ when $D_{\infty}=\{(\xi, I[N])\}$ is a $\gamma_{k}$-fine partial division of $\mathbb{R}(0,1]$ with $\xi \in \overline{\mathbb{R}}^{(0,1]}, \mathbb{R}^{(0,1]}$.

Let $K>\frac{1}{\epsilon}$ be a positive integer and $\gamma=\gamma_{K}$. Let $D$ be a $\gamma$-fine division of $\mathbb{R}^{(0,1]}, D_{\infty}=\left\{\left(\xi, I[N]: \xi \in \overline{\mathbb{R}}^{(0,1]}, \mathbb{R}^{(0,1]}\right\}\right.$ and $D_{1}=D, D_{\infty}$. Then, for any $(\xi, I[N]) \in D_{1} \cap \Gamma_{1 / K}^{c}$, we have

$$
|f(\xi) G(I[N])-F(I[N])| \leq \frac{1}{K} G(I[N])<\epsilon G(I[N]),
$$

i.e., 


$$
\left(D_{1}\right) \sum_{(\xi, I N]] \notin \Gamma_{1 / K}}|f(\xi) G(I[N])-F(I[N])|<\epsilon \cdot(D) \sum_{(\xi,[N]) \notin \Gamma_{1 / K} / K} G(I[N]) .
$$

Thus, we have

$$
\begin{aligned}
\left|(D) \sum f(\xi) G(I[N])-F\left(\mathbb{R}^{(0,1]}\right)\right|= \\
\left|(D) \sum(f(\xi) G(I[N])-F(I[N]))\right| \\
\leq\left(D_{1}\right) \sum_{(\xi, I N]) \in \Gamma_{1 / k}}|f(\xi) G(I[N])|+\left(D_{1}\right) \\
\quad \sum_{(\xi, I N]) \in \Gamma_{1 / K}|F(I[N])|+\left(D_{1}\right)}\left|\sum_{(\xi, I N]) \notin \Gamma_{1 / K}}\right| f(\xi) G(I[N])-F(I[N]) \mid \\
+\left(D_{\infty}\right) \sum|F(I[N])| \leq \epsilon+\epsilon+\epsilon \cdot(D) \\
\quad \sum_{(\xi, I N] \notin \Gamma_{1 / K}} G(I[N])+\epsilon .
\end{aligned}
$$

Since there exists a constant $B$ such that $(D) \sum G(I[N])$ $\leq B$ for any $D$ (Muldowney 2012, p. 285, Theorem 168), we get the desired result.

The following theorem is a consequence of Theorem 2 .

Theorem 3 Let $f: \overline{\mathbb{R}}^{(0,1]} \rightarrow \mathbb{R}$ and $F: \mathrm{I} \rightarrow \mathbb{R}$ be additive. Then $f$ is Henstock-Wiener integrable on $\mathbb{R}^{(0,1]}$ with primitive $F$ if and only if $F$ is $A C$ near infinity and for each $k$, $k=1,2, \ldots$, we have $I V\left(h, \Gamma_{1 / k}\right)=0$ and $I V\left(F, \Gamma_{1 / k}\right)=0$.

The following theorem follows immediately from Theorem 3.

Theorem 4 Let $f: \overline{\mathbb{R}}^{(0,1]} \rightarrow \mathbb{R}$ and $F: I \rightarrow \mathbb{R}$ be additive. Then $f$ is Henstock-Wiener integrable on $\mathbb{R}^{(0,1]}$ with primitive $F$ if and only if $F$ is $A C$ near infinity and $F^{\prime}(I[N])=f(\xi)$ almost all $(\xi, I[N])$ in $\mathbb{R}^{(0,1]} \times \mathrm{I}$.

\section{ACKNOWLEDGEMENTS}

The first author of this research was supported by the Faculty of Science Research Fund, Prince of Songkla University, Hat Yai, Thailand.

\section{REFERENCES}

Apostol, T.M. 1957. Mathematical Analysis. Boston: AddisonWesley.

Cabral, E. \& Lee, P.Y. 2000-2001. A fundamental theorem of calculus for the Kurzweil-Henstock integrals in $\mathbb{R}^{m}$. Real Analysis Exchange 26: 867-876.

Cameron, R.H. \& Martin, W.T. 1944. Transformations of Wiener integrals under translations. Annals of Mathematics 45(2): 386-396.

Chew, T.S. \& Lee, P.Y. 1994. The Henstock-Wiener integral. Proceeding of Symposium on Real Analysis (Xiamen 1993). J. Math. Study 27(1): 60-65.

Henstock, R. 1988. Lectures on the Theory of Integration. Singapore: World Scientific.

Kurzweil, J. 2000. Henstock-Kurzweil Integration: Its Relation to Topological Vector Spaces. Singapore: World Scientific.

Lee, P.Y. \& Výborný, R. 2000. The Integral: An Easy Approach after Kurzweil and Henstock. Cambridge: Cambridge University Press.

Lu, J. \& Lee, P.Y. 1999. The primitive of Henstock integrable functions in Euclidean space. Bull. London Math. Society 31: 173-180.

Muldowney, P. 2012. A Modern Theory of Random Variation With Applications in Stochastic Calculus, Financial Mathematics, and Feynman Integration. New York: John Wiley \& Sons.

Muldowney, P. 1987. A General Theory of Integration in Function Spaces, including Wiener and Feynman Integration. Pitman Research Notes in Mathematics Series. Harlow: Longman.

Yang, C.H. 1998. Measure theory and the Henstock-Wiener integral. M.Sc. Thesis. NUS (unpublished).

Yang, C.H. \& Chew, T.S. 1999. On McShane-Wiener integral. Proceeding of International Mathematics Conference (Manila 1998), Matimyas Math. 22(2): 39-46.

Varayu Boonpogkrong*

Department of Mathematics and Statistics

Faculty of Science

Prince of Songkla University

Hat Yai, 90112

Thailand

Elvira Pederes de Lara-Tuprio

Mathematics Department

Ateneo de Manila University Loyola Hts.

Quezon City, 1108

Philippines

*Corresponding author; email: varayu.b@psu.ac.th

Received: 8 November 2016

Accepted: 18 April 2017 